\title{
Brain XQTL map: integrating the genetic architecture of the human brain transcriptome and epigenome
}

\author{
$\mathrm{Ng} \mathrm{B}^{1,2}$, White $\mathrm{CC}^{3}$, Klein $\mathrm{H}^{3,4}$, Sieberts $\mathrm{SK}^{5}$, McCabe $\mathrm{C}^{3}$, Patrick $\mathrm{E}^{3}, \mathrm{Xu} \mathrm{J}^{3}$, Yu L ${ }^{6}$, \\ Gaiteri $\mathrm{C}^{6}$, Bennett $\mathrm{DA}^{6}$, Mostafavi $\mathrm{S}^{1,2, *}$, , De Jager $\mathrm{PL}^{3,4, * \#}$ \\ ${ }^{1}$ Department of Statistics and Department of Medical Genetics, University of British Columbia, Vancouver, \\ British Columbia, Canada. \\ ${ }^{2}$ Centre for Molecular Medicine and Therapeutics, Vancouver, British Columbia, Canada. \\ ${ }^{3}$ Broad Institute, Cambridge, Massachusetts, USA. \\ ${ }^{4}$ Center for Translational \& Systems Neuroimmunology, Department of Neurology, Columbia University \\ Medical Center, New York, New York, USA. \\ ${ }^{5}$ Sage Bionetworks, Seattle, Washington, USA. \\ ${ }^{6}$ Rush Alzheimer's Disease Center, Rush University Medical Center, Chicago, Illinois, USA. \\ \# contributed equally \\ *To whom the correspondence should be addressed to: pdejager@rics.bwh.harvard.edu and \\ saram@stat.ubc.ca
}




\begin{abstract}
We perform quantitative trait locus (xQTL) analyses on a multi-omic dataset, comprising RNA sequence, DNA methylation, and histone acetylation ChIP sequence data from the dorsolateral prefrontal cortex of 411 older adult individuals. We identify SNPs that are significantly associated with gene expression, DNA methylation, and histone modification levels. Many SNPs influence more than one type of molecular feature, and epigenetic features are shown to mediate eQTLs in a number of $(9 \%)$ such loci. We illustrate the utility of our new resource, xQTL Serve, in prioritizing the cell type most affected by an $x Q T L$ and in enhancing genome wide association studies (GWAS) as we report 18 additional CNS disease susceptibility loci after re-analyzing published studies.
\end{abstract}




\section{Introduction}

Genome wide association studies (GWAS) have identified thousands of SNPs that are associated with various human disease ${ }^{1}$. However, the majority of identified SNPs fall in the non-coding regions of the genome ${ }^{2}$. Connecting these regulatory changes to specific genes or to molecular pathways that may be implicated in human diseases is not straightforward. Suggestive evidence indicate that many more such SNPs exist, but they are difficult to detect due to their typically small effect sizes and the challenge of multiple testing burden in genome-wide assessment of common genetic variation ${ }^{3}$.

Expression quantitative trait locus (eQTL) analyses ${ }^{4-9}$ have been very useful in understanding the functional consequences of trait- and disease-associated variants and in identifying genes that are likely to be affected by a risk allele. Recently, QTL analyses have been extended to other molecular phenotypes, such as DNA methylation $(\mathrm{mQTL})^{10,11}$ and histone modification $(\mathrm{haQTL})^{12}$. Overall, SNPs associated with molecular phenotypes (xQTLs) are over-represented among SNPs that are linked to various traits and diseases ${ }^{8,13}$, and previous studies have used eQTL hits to prioritize associations in GWAS, leading to improved detection sensitivity ${ }^{14-16}$. While a few datasets exist for brain tissue, large datasets measuring all three of these epigenomic and transcriptomic features have only recently been generated from the same brain region of the same individuals.

Here, we present a new Resource for the neuroscience community by performing $x Q T L$ analyses on a multi-omic dataset that consists of RNA sequence (RNA-seq), DNA methylation, and histone acetylation (H3K9Ac ChIP-seq) data derived from the dorsolateral prefrontal cortex (DLPFC) of up to 494 subjects (411 subjects having all three data types available). Samples are collected from participants of the Religious Orders Study (ROS) and the Rush Memory and Aging Project (MAP), which are two longitudinal studies of aging designed by the same group of investigators. These studies share the same sample and data collection procedures, which naturally permits joint analyses ${ }^{17,18}$. At its heart, the Resource presents a list of SNPs associated with cortical gene expression, DNA methylation, and/or histone modification levels that reflects the impact of genetic variation on the transcriptome and epigenome of aging brains. While our XQTLs replicated well in both brain and blood, a notable portion is specific to genes that are only expressed in brain. Also, many SNPs influence multiple molecular features, with a small number of them having their impacts on gene expression mediated through epigenetics. Further, we apply a computational approach to prioritize the cell types that may be driving the tissue-level effect, a critical piece of information for informing the design of follow-up molecular experiments in which an in vitro or in vivo target cell type needs to be selected. Finally, we illustrate the efficacy of an "xQTL-weighted GWAS" approach for applying our xQTLs to improve the statistical power of GWAS, and we identify a number of additional susceptibility variants for 
several diseases. All data used in this study are available from www.radc.rush.edu, and the $x Q T L$ results and analysis scripts can be accessed through our online portal, $\mathbf{x Q T L}$ Serve, at http://mostafavilab.stat.ubc.ca/xQTLServe.

\section{Results}

$x Q T L$ Discovery

Genotype data ${ }^{19}$ were generated from 2,093 individuals of European-descent. Of these individuals, gene expression (RNA-seq) $(n=494)$, DNA methylation ${ }^{20}$ (450K Illumina array $)(n=468)$, and histone modification data (H3K9Ac ChIP-seq) $(n=433)$ were derived from post-mortem frozen samples of a single cortical region, the dorsolateral prefrontal cortex (DLPFC) (Figure 1A). 411 individuals have all four data types. Demographics of the analyzed individuals are summarized in Tables S1. Although some of these data have been previously published with respect to analysis of aging brain phenotypes (see Table S2), here we report genome-wide $x Q T L$ analyses for these datasets for the first time. Genotype imputation was performed using BEAGLE 3.3.2 ${ }^{21}$ with the 1000 genome reference panel $^{22}$, yielding 7,321,515 SNPs for analysis. For the molecular phenotype data, 13,484 expressed genes, 420,103 methylation sites, and 26,384 acetylation peaks remained after quality control (QC) analyses. The effects of known and hidden confounding factors were removed from the molecular phenotype data using linear regression (Supplementary Information). Consistent with previous studies, we observed that accounting for hidden confounding factors greatly enhances the statistical power of cis eQTL detection ${ }^{23,24}$, and we confirm that this observation holds true for cis $\mathrm{mQTL}$ and cis haQTL detection (Figure S1).

We employed Spearman's rank correlation to estimate the association strength between alleles of each SNP and gene expression, DNA methylation, and histone acetylation levels. We refer to the measurement unit of each molecular phenotype data as a feature and a significant association between a SNP and a feature as an $\times Q T L$ (i.e. an $x Q T L$ is a SNP-feature pair). Based on the results of prior studies, we performed cis $x Q T L$ analysis between SNPs and each feature by defining a window size of $1 \mathrm{Mb}$ for eQTL analysis and haQTL analysis, and a $5 \mathrm{~Kb}$ window for $\mathrm{mQTL}$ analysis ${ }^{25-27}$. The $1 \mathrm{Mb}$ window for haQTL analysis was motivated by the possibility that SNPs in enhancer regions, which are far away, can indeed impact gene regulation through interaction (e.g. chromatin looping) with promoter regions. The much smaller window for the $\mathrm{mQTL}$ analysis was selected since the majority of cis mQTLs with the strongest correlation lie within a window of this size ${ }^{27,28}$. Also, the smaller window size helps reduce the multiple testing burden, given the much larger number of DNA methylation features. 
Using a Bonferroni corrected p-value threshold $\left(\alpha_{\text {FWER }}=0.05\right)$, we found $(1) 3,388$ genes associated with eQTL SNPs $\left(p<8 \times 10^{-10}\right)$, (2) 56,973 CG dinucleotides linked to mQTL SNPs $\left(p<5 \times 10^{-9}\right)$, and $1,681 \mathrm{H} 3 \mathrm{~K} 9 A \mathrm{Ac}$ peaks influenced by haQTL SNPs $\left(p<4 \times 10^{-}\right.$ ${ }^{10}$ ) (Figure 1B-C, Table 1). Among the eQTL genes, 133 of them correspond to lincRNAs out of a total of 391 lincRNAs tested. For results based on several other (cis) window sizes, see Supplementary Information. The complete lists of eQTLs, mQTLs, and haQTLs are provided through the XQTL Serve webpage (http://mostafavilab.stat.ubc.ca/xQTLServe).

\section{Replication and cross-tissue comparisons}

We evaluated the extent to which our $x Q T L s$ replicate eQTLs and $m Q T L s$ found in prior studies. We focused on $\mathrm{eQTL}$ and $\mathrm{mQTL}$ replication since relevant large-sample datasets are only available for these two $x Q T L$ types. We assessed the replication rate of eQTLs and mQTLs discovered in these studies in our dataset using the $\pi_{1}$ statistics ${ }^{32}$, which estimates the proportion of these eQTLs (mQTLs) that are also significant in our dataset. $\pi_{1}$ of the eQTLs are 0.91 and 0.56 for CommonMind and Braineac, respectively, and $\pi_{1}$ of mQTLs is 0.87 , which are all greater than their respective empirical null mean of 0.11 and 0.33 for eQTLs and mQTLs, respectively $(p<0.0001$, see Supplementary Information). The lower replication rate of Braineac eQTLs compared to CommonMind eQTLs could be due to its smaller sample size. Also, the Braineac eQTLs are based on false discovery rate (FDR) correction whereas CommonMind eQTLs were defined using Bonferroni correction, and stronger associations captured by more stringent correction are more likely to replicate ${ }^{33}$. In the reverse direction, we also assessed the replication rate of our eQTLs in the commonMind data, and estimated similar replication rate $\left(\pi_{1}=0.90\right)$. For the $\mathrm{mQTL}$ replication analysis, we explored restricting our $\mathrm{mQTL}$ analysis to a $100 \mathrm{~Kb}$ window, and observed similar replication rate $\left(\pi_{1}=0.87\right)$ on the fetal brain mQTLs, which suggests a $5 \mathrm{~Kb}$ window already captures majority of the stronger associations between SNP and DNA methylation.

For assessing cross-tissue replication, we used a large whole-blood eQTL dataset from the Depression Genes and Networks (DGN) ${ }^{33}$ study comprising 922 individuals of European descent between 21 to 60 years old and two smaller eQTL datasets from the Immune Variation (ImmVar) study ${ }^{34}$ that consist of monocyte and T cell data from 211 individuals of European descent between 18 to 50 years old. $\pi_{1}$ of these eQTLs in our dataset are 0.63 (whole blood), 0.61 (monocytes), and 0.67 ( $T$ cells), which are greater than their empirical null mean of 0.10 ( $p<0.0001$ for all three datasets). Thus, a large proportion of blood eQTLs are present in our brain data. Since blood contains a mixture of cell types including immune cells that share characteristics with those in brain, we 
also assessed the replication rate on three additional tissues, namely, subcutaneous adipose, visceral adipose, and liver from the GTEx study ${ }^{35}$. The replication rates are $0.51,0.38$, and 0.20 , respectively, which are indeed lower than that of blood.

Since DGN is one the largest and hence most well-powered eQTL studies, we also assessed flipping the role of the datasets in the $\pi_{1}$ estimation here. Specifically, we assessed the replication rate of our brain-derived eQTLs in the whole-blood DGN dataset (Figure 2A-B). When we consider SNP-gene pairs that can be tested in both studies, we observed a replication rate of 0.83 (Figure $2 \mathrm{C}$ ), which is greater than its empirical null mean of 0.30 ( $p<0.0001)$. This increase in replication rate when assessing eQTLs from our brain study in the DGN dataset may be due to the higher statistical power of the DGN study ( $n=922$ in DGN study and $n=494$ in ROSMAP study) and the fact that cortical tissue consists of a large variety of cell types which, in aggregate, express a large proportion of the transcriptome. Additional replication results for different tissues, window sizes, and XQTL types are provided in Table S3.

An important question to answer with our data is whether and which of the detected $x Q T L s$ are brain-specific. However, without tissue samples from the same individuals, distinguishing between subject-specific and tissue-specific effects is not possible. Nonetheless, based on the sparsity of "population-specific" eQTLs ${ }^{34}$ and a lower replication rate of eQTLs in blood compared to brain, a notable fraction of our eQTLs are likely tissue-specific. For example, when we considered only eQTLs that consist of the top SNP for each gene, we found that, of the 2,416 eQTLs discovered in our cortical tissue study that are testable in the whole-blood dataset, 433 eQTLs (18\%) have an unadjusted $p$-value $>0.05$, indicating that this subset of brain eQTLs are unlikely to be present in blood (Figure 2B). As an example, NLRP1 is expressed in both brain and blood (whole blood, monocytes and T cells), but its expression is only associated with brain-specific eQTL SNPS (Figure 2D). NLRP1 is a member of the NLRP1 inflammasome complex that is implicated in inflammatory response in both immune cells (in particular myeloid cells) and in brain ${ }^{36}$. Interestingly, a few small-scale studies also linked polymorphisms in this gene with amyloid-beta secretion and Alzheimer's disease $(A D)^{37}$. In addition to the 2,416 eQTLs that are testable in both brain and blood, we identified 809 eQTL target genes from our brain analysis that were absent from the DGN's blood eQTL analysis because the corresponding genes were not expressed in blood. As expected, this set of 809 brain-specific eQTL genes are enriched for brainrelevant functions (GSEA enrichment analysis, FDR<0.05) such as "Neuronal System", "Potassium Channel Components", and "Neurotransmitter Receptor Binding".

Overall, the high cross-sample and cross-tissue replication rates suggest that a large number of SNPs that impact molecular phenotypes are likely shared across contexts. 
The degree of overlap between brain and blood eQTLs is quite high, with $a \pi_{1}$ of $\sim 0.8$. Nevertheless, our results suggest some eQTLs are tissue-specific, and more tissuespecific effects would likely emerge from analyses of purified cell populations.

Genetic architecture of $x Q T L$ SNPs and sharing across molecular phenotypes

We used genomic annotations based on DLPFC tissue from ChromHMM ${ }^{38}$ and computed the odds of an XQTL SNP belonging to 1 of 15 regulatory regions (annotated by chromatin states) as compared to all non-xQTL SNPs proximal to molecular features, i.e. within $1 \mathrm{Mb}, 5 \mathrm{~Kb}$, and $1 \mathrm{Mb}$ windows for $\mathrm{eQTL}$, $\mathrm{mQTL}$, and haQTL analyses with all SNPs tested in these analyses considered as proximal. As shown in Figure 3A, eQTL SNPs are mainly enriched in promoters and transcribed regions, conforming to our understanding of how SNPs at transcription factor (TF) binding sites can affect proteinDNA interactions ${ }^{39}$ and how SNPs in transcribed regions are known to affect mRNA processing and turnover ${ }^{40}$. haQTL SNPs are also largely enriched in promoter and transcribed regions, consistent with the role of H3K9Ac in transcriptional activation ${ }^{41}$. By contrast, mQTL SNPs are mainly enriched in bivalent regions (promoters and enhancers) and PolyComb repressed regions, which matches prior findings that a large portion of $\mathrm{mQTL}$ SNPs resides in chromatin regions that are developmentally regulated $^{27}$. Also, suppressed gene expression in PolyComb repressed regions might partly explain why eQTL and haQTL SNPs derived from adult samples are scarce in these regions. Notably, $x Q T L$ SNPs that are shared across all three molecular phenotypes are mainly enriched close to the TSS as well as in the 5' and 3' transcribed regions. With respect to transcribed sequences, we saw enrichment for all types of $x Q T L s$ in exons relative to introns (Figure 3B), with this trend being most striking for mQTLs.

To quantify the degree to which an $x Q T L$ SNP influences more than one molecular phenotype, we first identified the list of XQTL SNPs for a "discovery" phenotype and then estimated the $\pi_{1}$ statistics of the SNP-feature associations for a "test" phenotype that share the same $x Q T L$ SNPs. Since an $x Q T L$ SNP might be tested for association with multiple cis features, e.g. an $\mathrm{mQTL}$ SNP was, on average, tested for association with 18 gene expression levels, a decision on which SNP-feature associations to include in the $\pi_{1}$ estimation was necessary (see Supplementary Information). In particular, we examined the distance between each pair of "discovery" SNP and "test" feature, and found this distance to be a prime determinant of cross-phenotype sharing. For example, the strongest associated eQTL gene for each $\mathrm{MQTL}$ SNP is often the gene closest to the mQTL SNP (Figure 3C). Based on this observation, we estimated $\Pi_{1}$ to be $0.41-0.63$ when we considered only the closest feature to each $x Q T L$ SNP (Figure 3D). Also, we examined the effect of window size by restricting the haQTL 
analyses to $2 \mathrm{~Kb}, 40 \mathrm{~Kb}$, and $100 \mathrm{~Kb}$ windows as well as changing the eQTL and mQTL analysis window to $100 \mathrm{~Kb}$, and found negligible differences in our estimates of $x Q T L$ sharing (Table S4).

The availability of multi-omic data from the same individuals enabled us to go beyond "overlap analyses" (Figure 4A) and to investigate the cascading effect of genetic variation through the measured regulatory genomics layers. Specifically, we investigated whether the effect of a regulatory cis XQTL SNP is mechanistically mediated through its impact on epigenetic modification or gene expression using the casual inference test $(\mathrm{CIT})^{42}$. This analysis was performed on 10,897 xQTL SNPs (impacting 629 genes based on the eQTL analysis) that are associated with all three molecular phenotypes, as only such SNPs satisfy the precondition for mediation analysis. With this analysis, we distinguished between three models for propagation of information from genetic variation: 1) independent effects of a SNP on cis gene expression and the cis epigenetic landscape (independent model or IM), 2) a propagation path from SNP to gene expression via epigenetic modifications (epigenetic mediation model or EM), or 3) a propagation path from SNP to the epigenome (namely DNAm) via gene expression (transcription mediation model or TM) (Figure 4B).

Using Bonferroni correction with the CIT test, we observed that $9 \%$ of the association sets conform to the EM model, $3 \%$ conform to the TM model, $85 \%$ conform to IM, and the remaining 3\% could not be classified (Figure 4C, Table S5). As an example, an xQTL SNP ( $r$ 13015714) associated with Celiac disease (GWAS $p<10^{-8}$ ) was found to affect IL1RL1 gene expression $\left(p<10^{-11}\right)$, DNA methylation $\left(p<10^{-30}\right)$ and histone modification $\left(p<10^{-12}\right.$ ), but the impact of this SNP on gene expression appeared to be fully mediated by epigenetic modifications (Figure 4D-E), and thus this SNP conforms to the EM model. We additionally tested whether GWAS SNPs (downloaded from the GWAS catalog ${ }^{1}$ ) are prefrentially enriched for any of these models but did not find any model-specific enrichment.

A large fraction of the shared $x Q T L$ SNPs appear to affect gene expression directly. This result could be explained by: 1) epigenetic modification playing a passive role $^{26}$ where gene expression in fact lies upstream of epigenetic modification ( $3 \%$ based on the TM model), 2) regulation of gene expression being dependent on a more complex combination of epigenetic marks that are not measured in our subjects, and 3) artefactual decorrelation between the expression and epigenomic features due to technical or other factors. Thus, we should see these estimates for mediation as a minimum of true mediation: these may be the most robust subset of mediation events. The important message of these analyses is that mediation exists at a substantial number of loci and further work and data may be needed to uncover additional loci. 
Indeed, in line with this hypothesis, when we separately included only DNA methylation or histone modification into the model, we identified a smaller subset of association sets for which an effect on gene expression was fully explained by the epigenetic features: $3 \%$ for DNA methylation and $6 \%$ for histone modification. Thus, a complementary (nonredundant) combination of DNA methylation and histone acetylation seems to be required to capture the mediation effect, and adding other non-redundant epigenetic features would likely further enhance detection of this type of functional propagation.

\section{Enrichment of disease susceptibility SNPs among xQTL SNPS}

Studies have shown that SNPs associated with eQTLs are more likely to influence complex traits and disease susceptibility ${ }^{8,13}$. Here, we provide further support for this observation for eQTLs, mQTLs, and haQTLs by performing an enrichment analysis on reported $p$-values of 16 GWAS datasets, including large-scale GWAS meta-analyses of $\mathrm{AD}^{43}$, Schizophrenia ${ }^{44}$, and type II Diabetes ${ }^{45}$ (Supplementary Information). Enrichment was assessed using stratified linkage disequilibrium (LD) score regression (LDSR) ${ }^{46}$. For all 12 GWAS studies (out of 16) with over 20,000 samples (Table S6, Figure 5A), significant enrichment was observed for the $x Q T L$ SNPs. We also repeated this analysis using a more stringent background model, where we considered enrichment of our xQTLs against a background set of SNPs falling in "generic" annotation categories as provided in the LDSR software ${ }^{46}$. Again, significant enrichment, albeit with lower effect size, was observed for many of the GWAS studies (Figure 5A, Table S6). Next, we hypothesized that SNPs shared between $x Q T L$ types, which affect multiple molecular phenotypes, are more likely to impact downstream processes and could constitute a list of "high confidence" functional SNPs. We therefore compared all $x Q T L$ SNPs that are shared across at least two molecular traits, against those xQTLs that are only found for one molecular trait. Indeed, we observed enrichment for the shared $x Q T L s$, but their enrichment was not always higher than the background $x Q T L$ SNPs, i.e. somewhat trait dependent (Table S6). To test the robustness of the results to window size, we repeated the analysis with $100 \mathrm{~Kb}$ windows for all three XQTL types (Table S7). The overall trend remained the same with slightly higher enrichment observed.

The enrichment results are reassuring, and, as we describe later, we can use our list of $x Q T L$ SNPs to prioritize testing in GWAS studies and identify new susceptibility loci. Also, investigators can use our XQTL list to annotate GWAS SNPs related to the brain or nervous system, which accelerates the transition to functional studies. For example, we used our eQTLs to map the 21 SNPs (and correlated SNPs in LD with $r^{2}>0.8$ ) reported in the IGAP AD GWAS and identified four candidate AD genes that are absent from the reported gene list defined by proximity ${ }^{43}$ (MADD, MTCH2, PILRA, and POLR2E). The TSS of these eQTL mapped genes were $>100 \mathrm{~Kb}$, on average, from their 
respective AD SNPs. MTCH2, PILRA, and POLR2E have also been found in recent eQTL mapping studies ${ }^{47}$, demonstrating the robustness of our results. MADD has not been previously reported in this context but is a good candidate given that its expression correlates with neuronal cell death in $\mathrm{AD}^{48}$ and that it has also been reported to modulate AD-related tau toxicity in a Drosophila model ${ }^{49}$.

Accelerating the transition to functional studies in specific cell types

Identification of the relevant cell type to target in vitro or in vivo functional studies is a major challenge since our $x Q T L$ study, like many others, relies on tissue profiles generated from a complex mixture of cell types. To help prioritize cell types for such follow-up efforts, we repeated the analyses relating each SNP to a given molecular feature but additionally included a variable that estimates the proportion of a cell type in the profiled tissue and an interaction term to identify those SNPs whose effects depend on the proportion of a target cell (Supplementary Information). This approach was recently validated using whole-blood data ${ }^{50}$.

Using eQTL results as an example, we examined the potential specificity of each lead eQTL SNP for five cell types that are abundant in the cortex: neurons, microglia cells, astrocytes, oligodendrocytes, and endothelial cells. With this approach ${ }^{50}$, we found that assignment to a single cell type remains ambiguous for most eQTLs (all cell-specificity p-values are available at http://mostafavilab.stat.ubc.ca/xQTLServe). In a minority of cases, our analysis returned an unambiguous result for the lead eQTL. For example, at an FDR <0.05 threshold, we identified 6 significant cell-specific eQTLs (1 astrocytic, 3 microglia, and 1 neuronal). One of these results is presented in Figure $5 \mathrm{C}$ : the $C P V L$ locus harbors an eQTL effect (rs11971828) that is stronger in microglial cells as demonstrated by the statistical interaction between the proportion of microglia and the genotype of the corresponding eQTL SNP. With a more lenient discovery strategy where we thresholded the interaction term at an FDR $<0.2$, we found putative cell-type specific effects in neurons $(n=13)$ and microglia $(n=22)$ (Figure 5B). At this significance level, microglia, which are present at low frequency in cortical tissue, show the most effects, probably because our approach reduces noise in the expression measures. As shown in Figure 5B, even though a small number of cell-specific eQTLs were identified using stringent multiple testing correction, our results can still be useful in prioritizing cell types for follow-up experiments, based on the observation that suggestive cell-type specific eQTL genes show clear cell type preferences. Many of these "top" cell-specific eQTL genes tend to conform to the expected function of the implicated cell. For example, the MGMT locus harbors an eQTL that ranks among the top 3 for oligodendrocytes-specificity $\left(p=1.5 \times 10^{-4}\right)$. This gene is known to play a role in oligodendrocyte function and its mutations are associated with oligodendrogliomas. 
These cell-specific results are intriguing but require molecular validation using purified cell populations from the cortex with matched genotype; these results should be seen as a way to prioritize the selection of genes and cell types to be validated in a given cell type.

$x Q T L-$ weighted GWAS for gene discovery efforts

Our large compendium of brain XQTLs can also be leveraged to accelerate gene discovery by boosting statistical power in GWAS. The simplest way of using our $x Q T L$ SNP list would be to restrict association analysis to our xQTL SNPs. However, such a strategy would miss other relevant SNPs that are not in our list (or were not tested in the cis $\mathrm{XQTL}$ analysis). Thus, we opted to use a weighted Bonferroni procedure ${ }^{51}$, which permits all SNPs to be analyzed but weights their $p$-values by their potential phenotypic relevance. We refer to this approach as an "xQTL-weighted GWAS". Provided that the weights are non-negative and average to one, strong control on family-wise error rate is guaranteed $^{51}$. We employed a binary weighting scheme, where $p$-values of $x Q T L S N P s$ were divided by $w_{1}$ and all other SNPs were divided by $w_{0}$ with $s=w_{1} / w_{0}>1$ (see Supplementary Information for $s$ selection). Consistent with the standard GWAS threshold, significance was declared at $p<5 \times 10^{-8}$. To not over-count the number of significant hits due to correlations between SNPs, we applied PLINK1.952 on the 1000 Genomes phase 1 data $^{22}$ to remove SNPs among the significant hits that are in linkage disequilibrium (LD) with one other $\left(r^{2}<0.2\right)$.

We compared four approaches: (1) no weighting, (2) weighting $x Q T L$ SNPs found for any of the molecular phenotypes, (3) weighting SNPs within predefined windows from the molecular features $(1 \mathrm{Mb}, 5 \mathrm{~Kb}$, and $1 \mathrm{Mb}$ for eQTL, mQTL, and haQTL analyses) to account for distance bias, (4) weighting generic functional SNP in the LDSR baseline model $^{46}$, and (5) weighting $\times Q T L$ SNPs that are shared across any of the molecular phenotypes. Over the 19 GWAS datasets (Supplementary Information), weighting xQTL SNPs resulted in equal or more GWAS hits than no weighting, except for inflammatory bowel disease (Table S8). For 8 of the 19 studies, the xQTL-weighted GWAS approach found at least 2 new independent loci (Table S8). By contrast, weighting SNPs within predefined windows from the molecular features $(1 \mathrm{Mb}, 5 \mathrm{~Kb}$, and $1 \mathrm{Mb}$ for eQTL, $\mathrm{mQTL}$, and haQTL, respectively) as well as weighting SNPs in the LDSR baseline model resulted in little change in detection sensitivity. Interestingly, the gain in sensitivity was not always the highest when we weighted the shared $x Q T L$ SNPs. Also, compared to weighting the DGN eQTL SNPs, weighting the union of all $x Q T L$ SNPs found in this study identified more additional independent susceptibility SNPs for a majority of the tested GWAS datasets, which demonstrates that additional signals are captured by mQTL and haQTL SNPs. In particular, weighting the xQTL SNPs found 22, 18, and 9 
additional independent SNPs for schizophrenia, height, and inflammatory bowel disease, respectively, compared to no weighting. In contrast, weighting the DGN eQTL SNPS found only 9, 3, and 2 additional independent SNPs. In fact, weighting just the ROSMAP eQTL SNPs identified 17 additional independent SNPs for schizophrenia, which illustrates the presence of eQTLs in our data that are enriched in brain diseases and not observed in blood.

Among the brain diseases that we examined, the largest detection gain was obtained with the schizophrenia dataset ${ }^{44}$, where 18 additional loci met genome-wide significance (excluding those near the MHC region) and were not in linkage disequilibrium (LD<0.2) with the reported susceptibility SNPs ${ }^{44} .7$ of these 18 SNPs were found to be associated with eQTLs (Table S8), including rs57709857, which influences LSM1, a gene previously found in a Han Chinese schizophrenia study ${ }^{53}$. However, the LSM1 locus had not reach genome-wide significance in individuals of European ancestry ${ }^{54}$. The list of eQTL genes also includes PCNX (associated with rs2189806), a member of the Notch signalling pathway that was reported to harbour a de novo copy number variant linked to Autism Spectrum Disorder ${ }^{55}$, and CPEB1 (associated with rs1864699), which was recently found to be implicated in experience-dependent neuronal development and circuit formation $^{56}$ (Figure 5C). Thus, several of our new schizophrenia loci have some face validity, but additional replication efforts are required to ensure that these are robust findings. In terms of the percentage increase in detection sensitivity, the largest gain was observed for Bipolar disorder ${ }^{57}$, where the standard GWAS approach identified one significant hit, whereas $\times Q T L-$ weighted GWAS identified 2 additional independent loci.

\section{Conclusion}

Using one of the largest multi-omic datasets for brain tissue, we generated a list of $x Q T L s$ as a Resource for the neuroscience community to further investigate the interplay between the genome, epigenome, and transcriptome in disease susceptibility. Our list of $x Q T L s$ replicates well in both brain and blood datasets, but it also contains $x Q T L s$ that appear to be unique to brain. Notable biological insights drawn from this Resource include the significant sharing of $x Q T L$ SNPs across the measured molecular phenotypes. Also, the effects of some eQTL SNPs are fully mediated by our two epigenetic features, and further work and data are needed to comprehensively address the extent to which epigenomic features mediate eQTL effects. Overall, we have created a large new reference with which investigators can functionally annotate their results, enhance their analyses, as illustrated by our xQTL-weighted GWAS approach, and guide further functional work, as with our cell type analysis. This Resource can be 
easily accessed through our portal, $\quad$ XTL $\quad$ Serve (http://mostafavilab.stat.ubc.ca/xQTLServe).

\section{Acknowledgements}

We thank the participants of ROS and MAP for their essential contributions and gift to these projects. This work has been supported by many different $\mathrm{NIH}$ grants: P330AG10161, U01 AG046152, R01AG16042, R01 AG036836, R01 AG015819, R01 AG017917, R01 AG036547. Data from these studies is available at the RADC Research Resource Sharing Hub at www.radc.rush.edu.

\section{Contributions}

Study design: SM, BN, PLD. Sample collection: DAB. Data generation and quality control analyses: BN, CM, HK, EP, JX, SM, PLD. Analyses: BN, CW, CG, SM. Interpretation of results and critical review of the manuscript: BN, CM, HK, EP, JX, CG, DAB, SM, PLD.

Table 1. Summary of $x Q T L$ associations.

\begin{tabular}{|c|c|c|c|c|c|c|}
\hline & \multicolumn{2}{|c|}{$\begin{array}{c}\text { No. associations (SNP-gene } \\
\text { pairs) }\end{array}$} & \multicolumn{2}{|c|}{ No. features } & \multicolumn{2}{|c|}{ No. SNPs } \\
\hline & Tested & Significant & Tested & Significant & Tested & Significant \\
\hline eQTLs (1Mb) & $60,456,556$ & 405,429 & 12,979 & 3,388 & $6,442,864$ & 313,467 \\
\hline mQTLs (5Kb) & $9,939,236$ & 693,696 & 412,152 & 56,973 & $2,358,873$ & 383,920 \\
\hline haQTLs (1Mb) & $125,100,450$ & 156,693 & 25,720 & 1,681 & $6,756,597$ & 119,778 \\
\hline
\end{tabular}

\section{Figure Legends}

Figure 1. Overview of XQTL analysis. (A) A graphical summary of data and analysis used in this study. (B) Manhattan plots, showing the negative - $\log _{10} p$-value ( $y$-axis) for association between a SNP and DNA methylation (mQTL), histone acetylation (haQTL) or gene expression (eQTL). The $x$ axis is the physical position in the genome. Each dot represents the strongest $p$-value (in a cis window) for each SNP. The bottom panel (C) shows the Manhattan plot for chromosome 18, to illustrate the distribution of $x Q T L s$ at a higher resolution. 
Figure 2. Cross-tissue replication analysis. (A) Scatter plot of the $-\log _{10} p$-values for association between the lead brain eQTL SNPs and their associated gene in brain and blood. The dashed red lines denote the significance threshold (FWER=0.05). (B) This panel zooms in on a small area of panel $A$ to display the distribution of eQTLs that appear to be brain-specific. (C) This panel shows the distribution of p-values of brain eQTLs when assessed in the DGN study. The estimated replication rate between blood and brain eQTLs, using the $\pi_{1}$ statistics, is 0.83 . (D) Here, we highlight one brainspecific eQTL by zooming in on the NLRP1 locus. Each dot represents one SNP tested in either the human cortex (ROSMAP, blue) or blood (DGN, pink). The $x$ axis represents the distance between each assessed cis SNP and the NLRP1 TSS, and the y-axis reports $-\log _{10} p$-values for association between SNPs and NLRP1 expression. The LD between the lead SNP in blood and brain is $r^{2}<0.1$.

Figure 3. Genomic enrichment of $x Q T L s$ and their overlap. (A) We present the log odds ratio of enrichment of XQTL SNPs in 15 different chromatin states as defined by the Roadmap Epigenomics project using data from two cognitively non-impaired ROSMAP and the ChromHMM algorithm. (B) This panel shows the enrichment of $x Q T L s$ in exons and introns. (C) For each lead $m Q T L S N P$, we computed its distance to the nearest TSS. The figure shows the distribution of distances to the TSSs from the lead mQTL SNPs. (D) $\pi_{1}$ statistics for cross-trait replication analysis: Each cell $(i, j)$ depicts the replication rate of SNPs identified in trait i ("discovery sample") when assessed in trait j ("replication sample"). In this replication setting, each discovery SNP was only assessed for association to its closest feature in the replication set.

Figure 4. Epigenetic mediation of eQTLs. (A) This panel shows a simple overlap analysis, to quantify the sharing between eQTL SNPs, mQTL SNPs, and haQTL SNPs. $2,305,942$ SNPs that are tested for all molecular phenotypes are considered in this analysis. (B) We illustrate the three models relating SNPs (s), epigenetic features (methylation/histone acetylation, $\mathrm{m} / \mathrm{h}$ ) and gene expression ( $\mathrm{g}$ ) that we investigated: (i) independent model (IM) where effects on epigenetic features and transcripts are unrelated, (ii) epigenetic mediation model (EM) where the epigenetic features mediate the SNP's effect on gene expression, and (iii) transcription mediation model (TM) where the effect of SNP on epigenetics is mediated through its effect on gene expression. (C) This plot presents the proportion of shared $x Q T L$ SNPs that are consistent with each of the models in panel B. (D) This box plot on the left shows the expression level of IL1RL1 as a function of the number of minor alleles present for rs13015714 (a shared $\mathrm{xQTL}$ SNP that impacts IL1RL1 and nearby DNA methylation and histone acetylation levels). The plot on the right shows that the SNP's effect disappears after regressing out the effect of the mQTL probe and haQTL peak associated with rs13015714 from expression of ILIRL1. (E) This panel shows the association between ILIRL1 and the 
levels of its associated methylation probe and acetylation peaks. Colors indicate the genotype for rs13015714: minor allele homozygotes (yellow), heterozygotes (green), major allele homozygotes (blue).

Figure 5. Application of the XQTL Resource for translational studies. (A) This bar plot shows enrichment of XQTL SNPs in several GWAS datasets based on the LDSR model (see Supplementary Information for GWAS references). The enrichments are with respect to two different sets of background SNPs: 1) all genome-wide SNPs and 2) SNPs falling in generic functional sites previously defined by LDSR. (B) This panel shows the $-\log _{10} p$-value for the interaction test (quantifying cell-specificity) for the 46 genes with an FDR $<0.2$. Colors indicate the level of significance of the interaction term, following the color key at the upper right aspect of the panel. (C) We illustrate the effect of changing proportions of microglia at the CPVL locus, plotting each subject's level of $C P V L$ expression in relation to a marker of microglial proportion (CD68 gene). The effect of the SNP's major allele on increasing CPVL expression increases as the proportion of microglia increases, particularly among major allele homozygotes (pink dots). (D-E) Zoomed in Manhattan plot around the PCNX (D) and CPEB1 (E) loci, showing the results of the published standard GWAS (bottom panel) and our weighted GWAS (top panel). Each dot is one SNP. The standard threshold of genome-wide significance $\left(p<5 \times 10^{-8}\right)$ is illustrated by a dotted green line. 


\section{Figure 1}

A

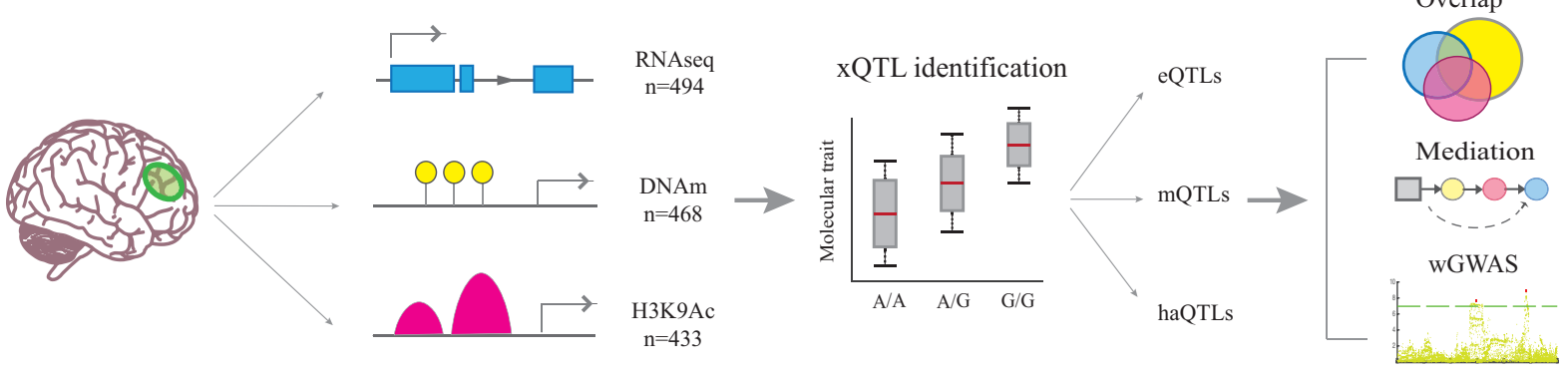

B
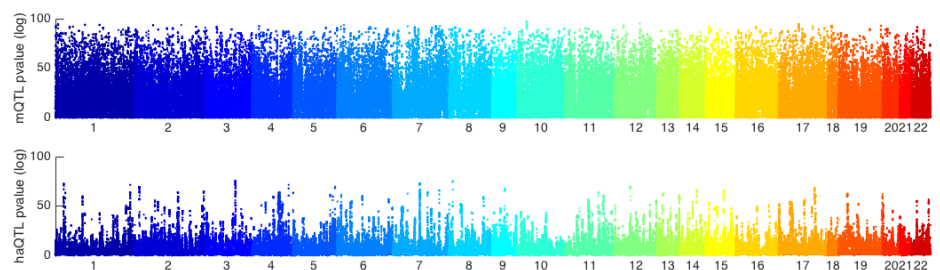

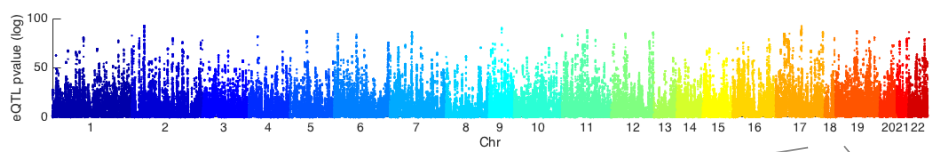

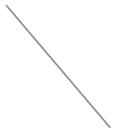

C
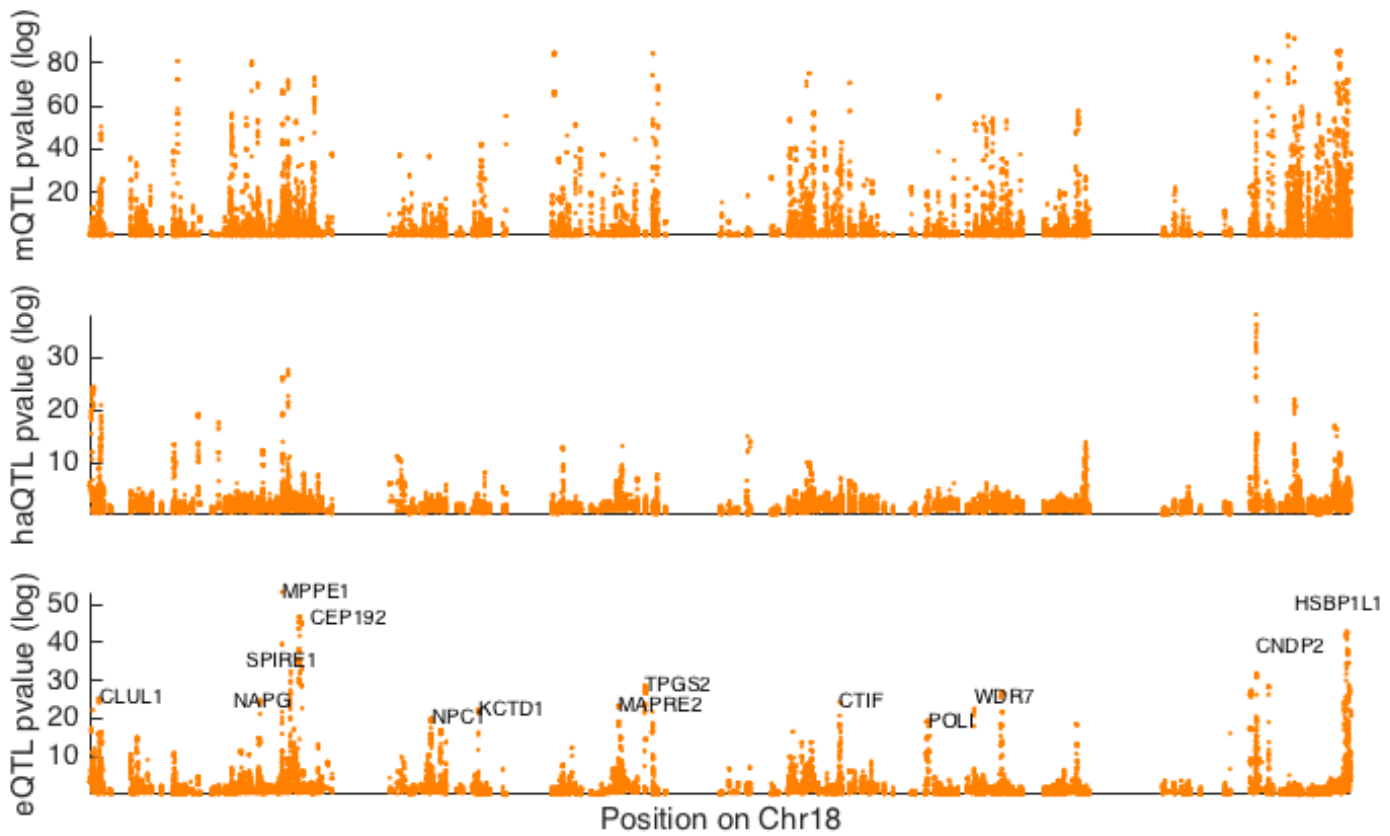
A

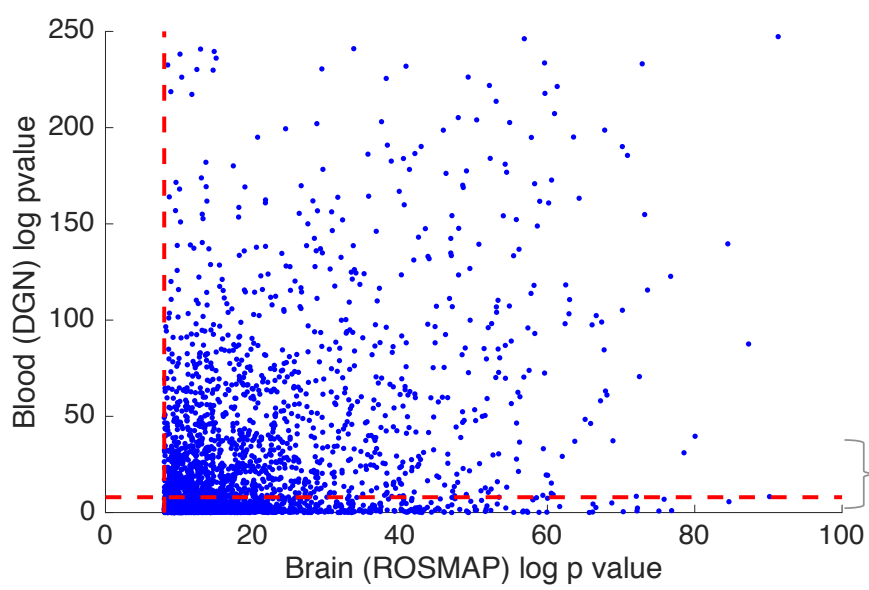

C

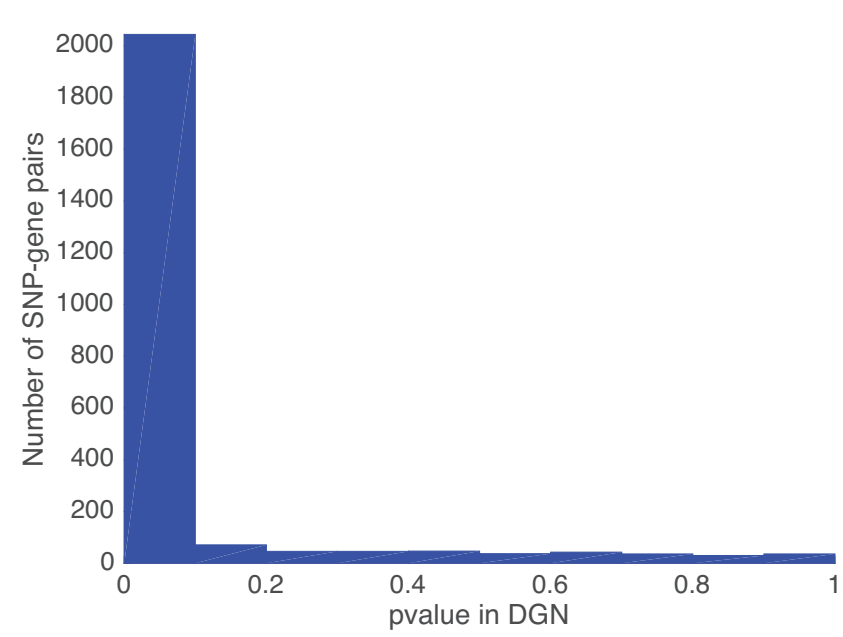

B

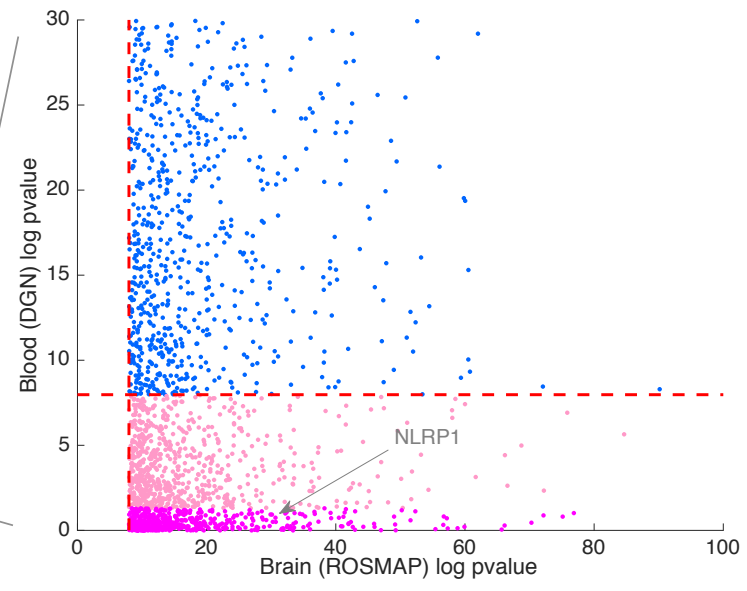

D

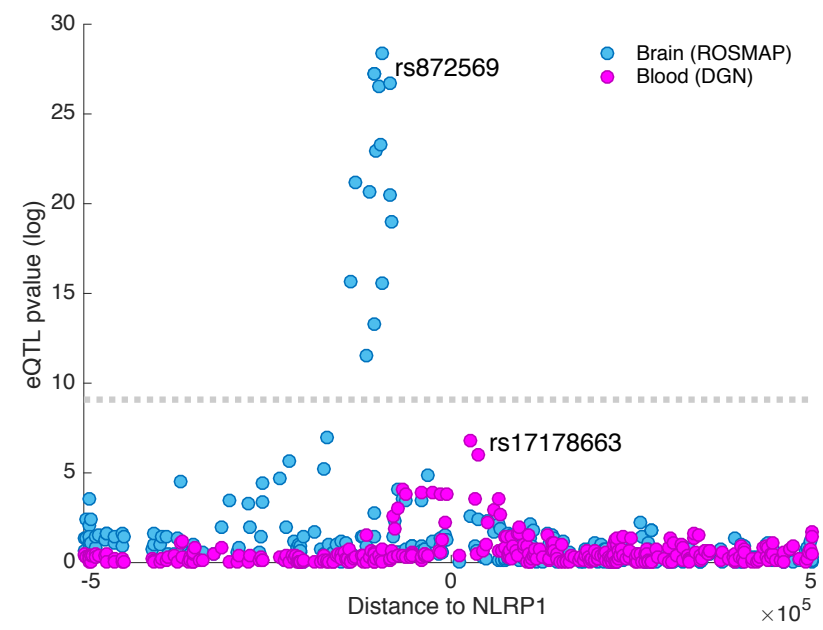


Figure 3

A

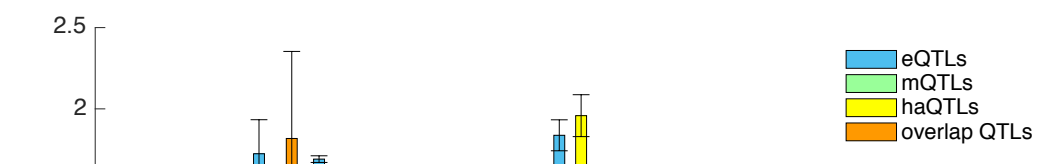

C

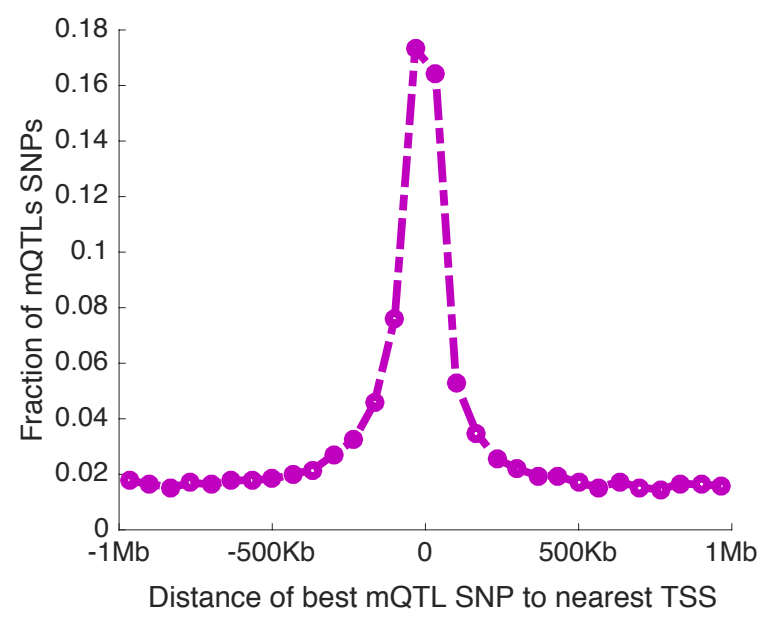

D
B

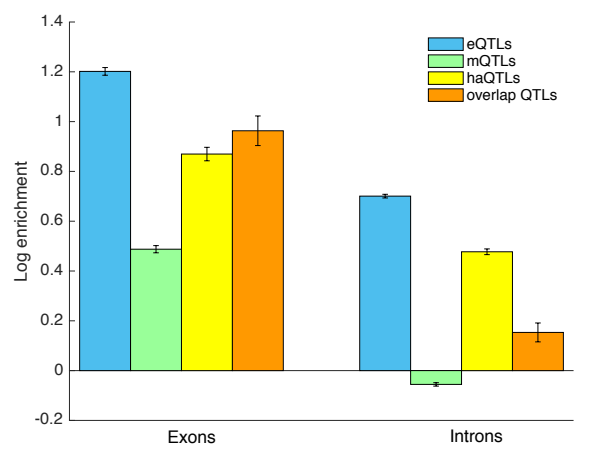

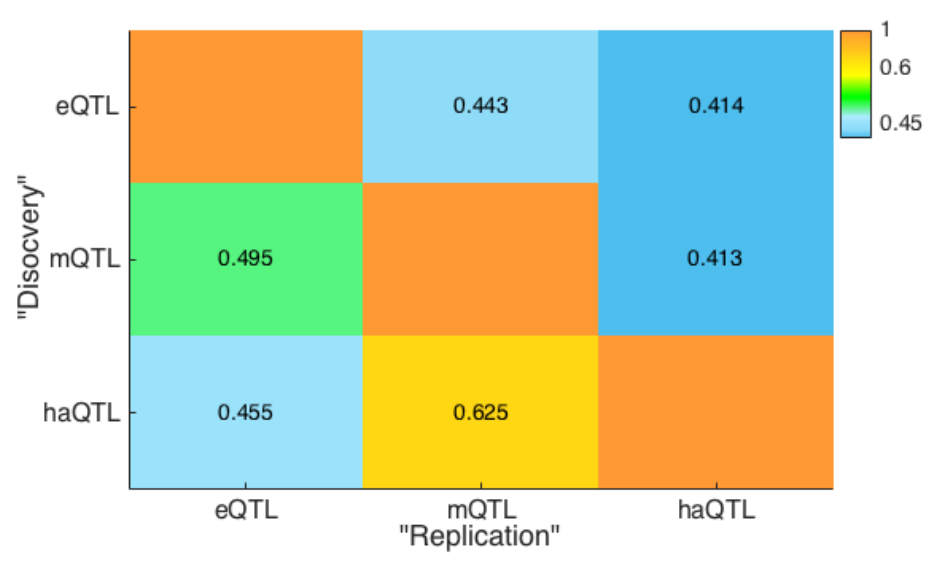


Figure 4

A

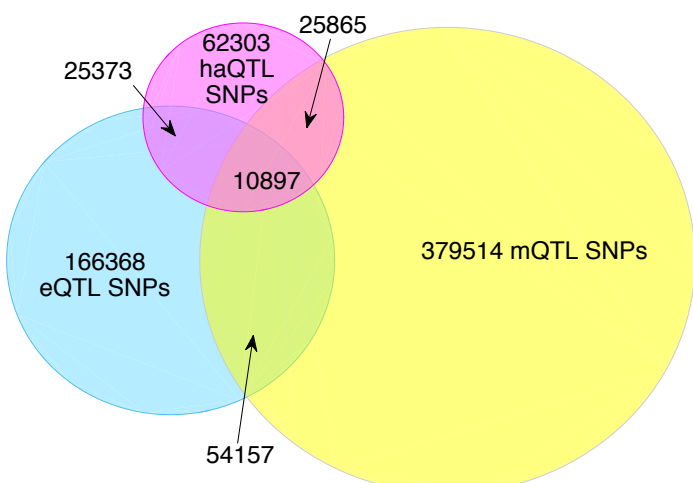

C

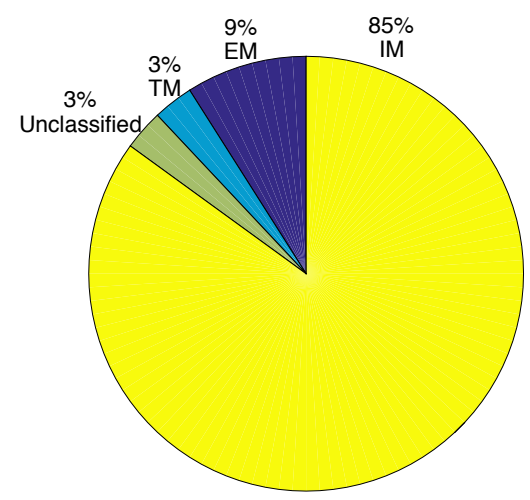

B

ii) $\mathrm{EM}$

iii) $\mathrm{TM}$
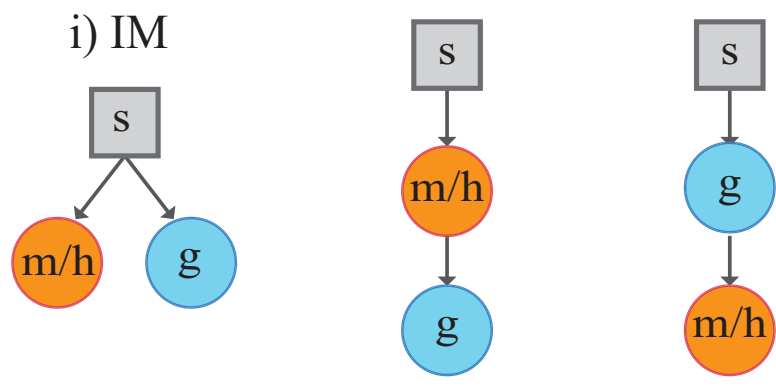

D
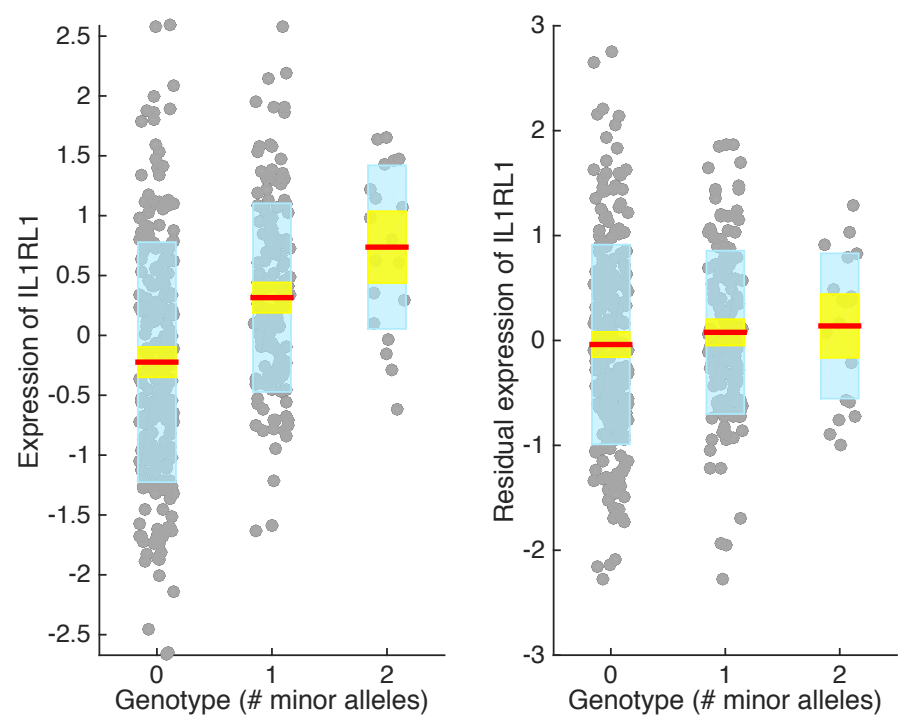

E
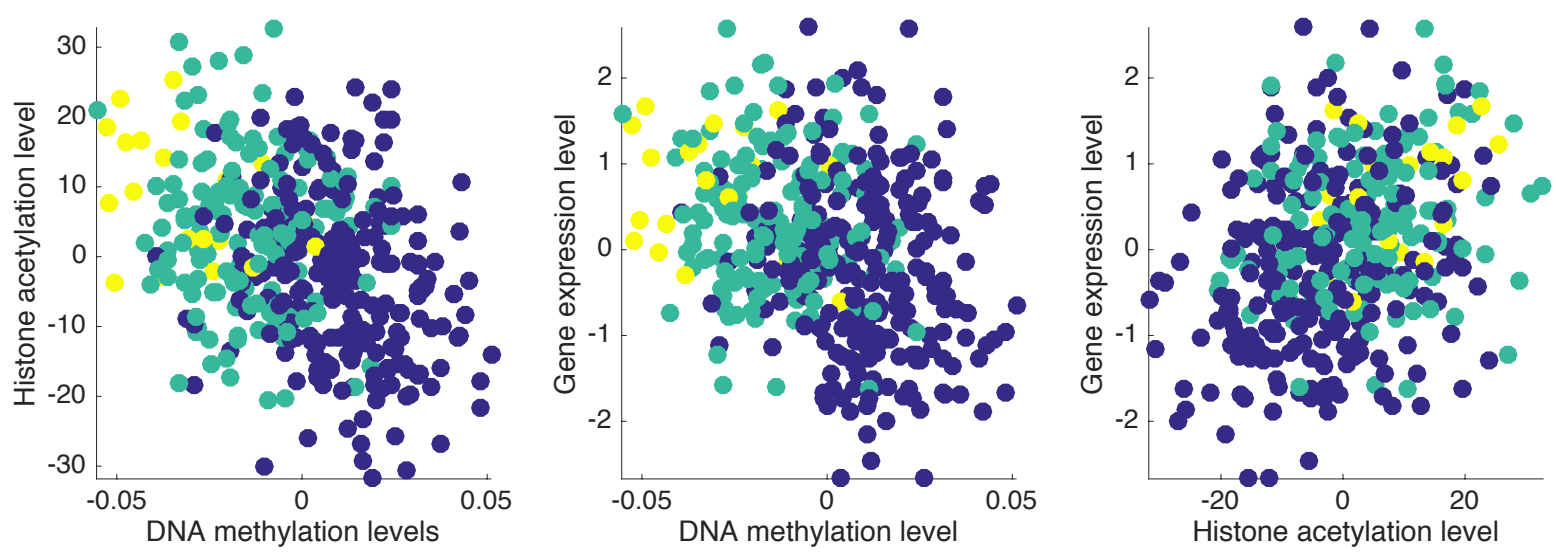


\section{Figure 5}

A

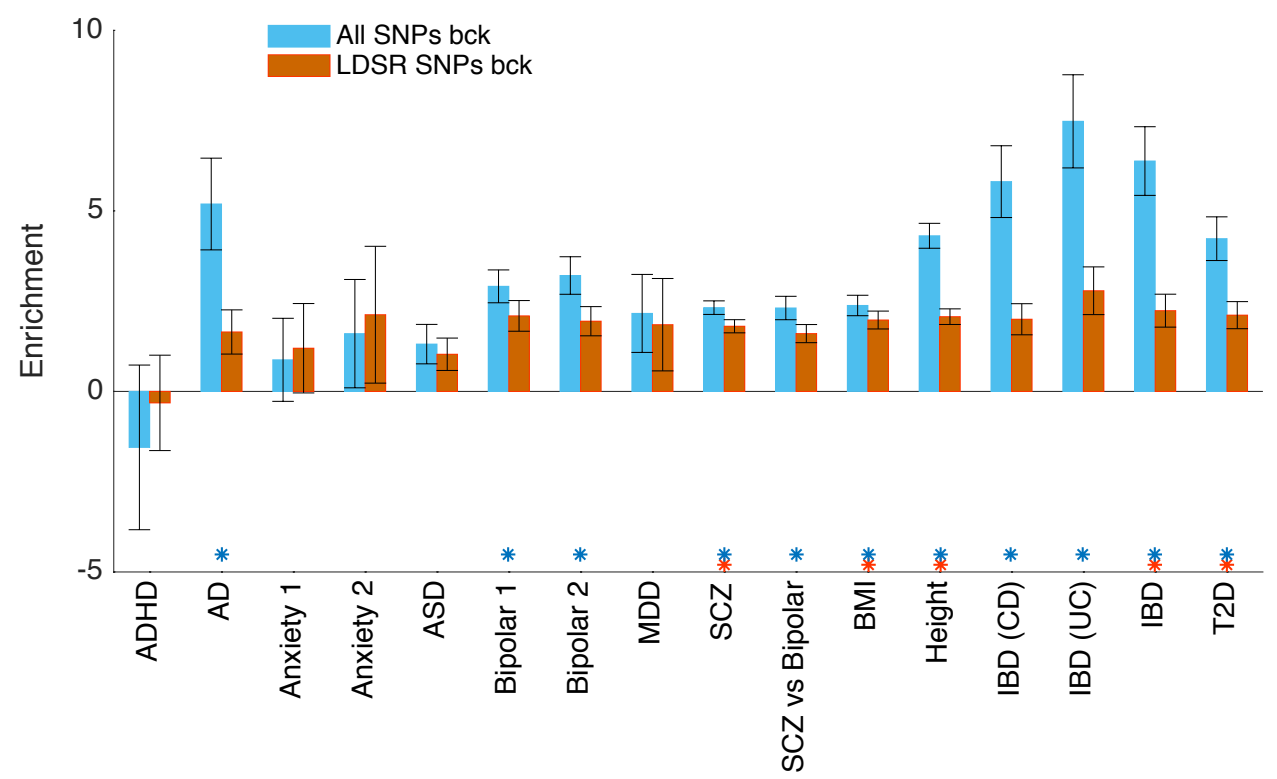

B

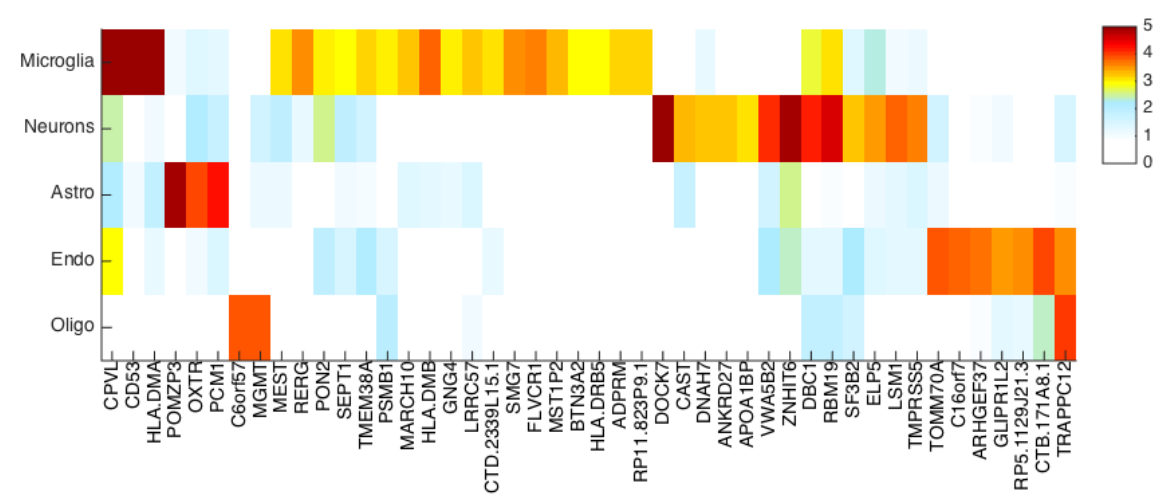

C

D
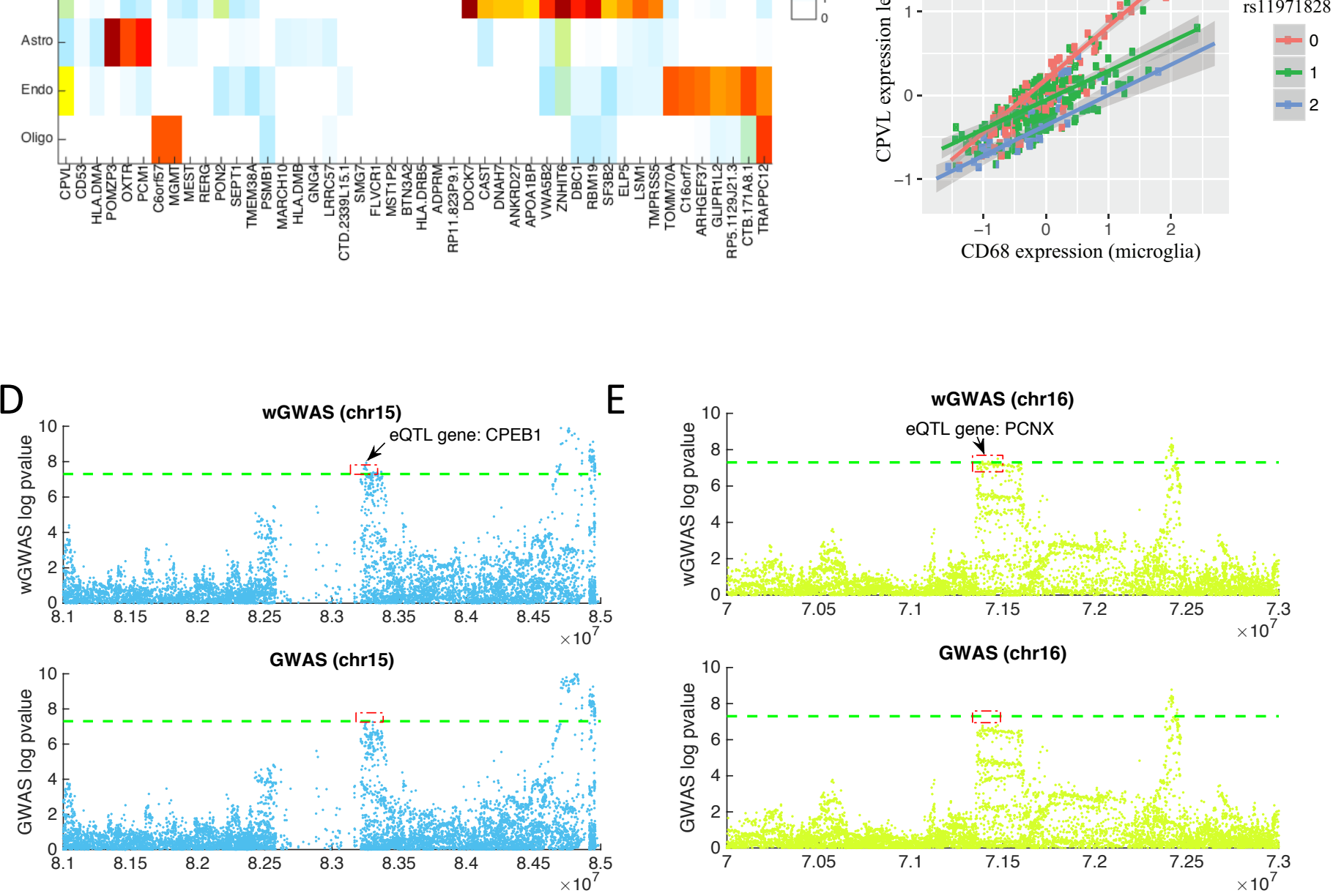


\section{References}

1 Welter, D. et al. The NHGRI GWAS Catalog, a curated resource of SNP-trait associations. Nucleic Acids Res 42, D1001-1006, doi:10.1093/nar/gkt1229 (2014).

2 Alexander, R. P., Fang, G., Rozowsky, J., Snyder, M. \& Gerstein, M. B. Annotating non-coding regions of the genome. Nat Rev Genet 11, 559-571, doi:10.1038/nrg2814 (2010).

3 Goldstein, D. B. Common genetic variation and human traits. New England Journal of Medicine 360, 1696 (2009).

4 Dimas, A. S. et al. Common regulatory variation impacts gene expression in a cell typedependent manner. Science 325, 1246-1250, doi:10.1126/science.1174148 (2009).

5 Montgomery, S. B. et al. Transcriptome genetics using second generation sequencing in a Caucasian population. Nature 464, 773-777, doi:10.1038/nature08903 (2010).

6 Pickrell, J. K. et al. Understanding mechanisms underlying human gene expression variation with RNA sequencing. Nature 464, 768-772, doi:10.1038/nature08872 (2010).

7 Lappalainen, T. et al. Transcriptome and genome sequencing uncovers functional variation in humans. Nature 501, 506-511, doi:10.1038/nature12531 (2013).

8 Human genomics. The Genotype-Tissue Expression (GTEx) pilot analysis: multitissue gene regulation in humans. Science 348, 648-660, doi:10.1126/science.1262110 (2015).

9 Brown, C. D., Mangravite, L. M. \& Engelhardt, B. E. Integrative modeling of eQTLs and cisregulatory elements suggests mechanisms underlying cell type specificity of eQTLs. PLoS Genet $\mathbf{9}$, e1003649, doi:10.1371/journal.pgen.1003649 (2013).

10 Gibbs, J. R. et al. Abundant quantitative trait loci exist for DNA methylation and gene expression in human brain. PLoS Genet 6, e1000952, doi:10.1371/journal.pgen.1000952 (2010).

11 Hannon, E. et al. Methylation QTLs in the developing brain and their enrichment in schizophrenia risk loci. Nat Neurosci 19, 48-54, doi:10.1038/nn.4182 (2016).

12 McVicker, G. et al. Identification of genetic variants that affect histone modifications in human cells. Science 342, 747-749, doi:10.1126/science.1242429 (2013).

13 Nicolae, D. L. et al. Trait-associated SNPs are more likely to be eQTLs: annotation to enhance discovery from GWAS. PLoS Genet 6, e1000888, doi:10.1371/journal.pgen.1000888 (2010).

14 Gamazon, E. R. et al. A gene-based association method for mapping traits using reference transcriptome data. Nat Genet 47, 1091-1098, doi:10.1038/ng.3367 (2015).

15 Ionita-Laza, I., McCallum, K., Xu, B. \& Buxbaum, J. D. A spectral approach integrating functional genomic annotations for coding and noncoding variants. Nat Genet 48, 214-220, doi:10.1038/ng.3477 (2016).

16 Zhu, Z. et al. Integration of summary data from GWAS and eQTL studies predicts complex trait gene targets. Nat Genet 48, 481-487, doi:10.1038/ng.3538 (2016).

17 Bennett, D. A., Schneider, J. A., Arvanitakis, Z. \& Wilson, R. S. Overview and findings from the religious orders study. Curr Alzheimer Res 9, 628-645 (2012).

18 Bennett, D. A. et al. Overview and findings from the rush Memory and Aging Project. Curr Alzheimer Res 9, 646-663 (2012).

19 De Jager, P. L. et al. A genome-wide scan for common variants affecting the rate of age-related cognitive decline. Neurobiol Aging 33, 1017 e1011-1015, doi:10.1016/j.neurobiolaging.2011.09.033 (2012).

20 De Jager, P. L. et al. Alzheimer's disease: early alterations in brain DNA methylation at ANK1, BIN1, RHBDF2 and other loci. Nature Neuroscience 17, 1156-1163 (2014).

21 Browning, B. L. \& Browning, S. R. A unified approach to genotype imputation and haplotypephase inference for large data sets of trios and unrelated individuals. Am J Hum Genet 84, 210223, doi:10.1016/j.ajhg.2009.01.005 (2009). 
Abecasis, G. R. et al. An integrated map of genetic variation from 1,092 human genomes. Nature 491, 56-65, doi:10.1038/nature11632 (2012).

23 Stegle, O., Parts, L., Durbin, R. \& Winn, J. A Bayesian framework to account for complex nongenetic factors in gene expression levels greatly increases power in eQTL studies. PLoS Comput Biol 6, e1000770 (2010).

24 Mostafavi, S. et al. Normalizing RNA-sequencing data by modeling hidden covariates with prior knowledge. PLoS One 8, e68141 (2013).

25 Banovich, N. E. et al. Methylation QTLs are associated with coordinated changes in transcription factor binding, histone modifications, and gene expression levels. PLoS Genet 10, e1004663, doi:10.1371/journal.pgen.1004663 (2014).

26 Gutierrez-Arcelus, M. et al. Passive and active DNA methylation and the interplay with genetic variation in gene regulation. Elife 2, e00523 (2013).

27 Do, C. et al. Mechanisms and Disease Associations of Haplotype-Dependent Allele-Specific DNA Methylation. Am J Hum Genet 98, 934-955, doi:10.1016/j.ajhg.2016.03.027 (2016). pedigrees. PLoS One 9, e99313, doi:10.1371/journal.pone.0099313 (2014). schizophrenia. Nat Neurosci 19, 1442-1453, doi:10.1038/nn.4399 (2016). human brain. Nat Neurosci 17, 1418-1428, doi:10.1038/nn.3801 (2014). Sun, W. et al. Histone Acetylome-wide Association Study of Autism Spectrum Disorder. Cell 167, 1385-1397 e1311, doi:10.1016/j.cell.2016.10.031 (2016).

32 Storey, J. D. \& Tibshirani, R. Statistical significance for genomewide studies. Proc Natl Acad Sci U S A 100, 9440-9445, doi:10.1073/pnas.1530509100 (2003).

33 Battle, A. et al. Characterizing the genetic basis of transcriptome diversity through RNAsequencing of 922 individuals. Genome research 24, 14-24 (2014).

34 Raj, T. et al. Polarization of the effects of autoimmune and neurodegenerative risk alleles in leukocytes. Science 344, 519-523, doi:10.1126/science.1249547 (2014).

35 The Genotype-Tissue Expression (GTEx) project. Nat Genet 45, 580-585, doi:10.1038/ng.2653 (2013).

36 Walsh, J. G., Muruve, D. A. \& Power, C. Inflammasomes in the CNS. Nat Rev Neurosci 15, 84-97, doi:10.1038/nrn3638 (2014).

37 Pontillo, A., Catamo, E., Arosio, B., Mari, D. \& Crovella, S. NALP1/NLRP1 genetic variants are associated with Alzheimer disease. Alzheimer Dis Assoc Disord 26, 277-281, doi:10.1097/WAD.0b013e318231a8ac (2012).

38 Ernst, J. \& Kellis, M. ChromHMM: automating chromatin-state discovery and characterization. Nat Methods 9, 215-216, doi:10.1038/nmeth.1906 (2012).

39 Gaffney, D. J. et al. Dissecting the regulatory architecture of gene expression QTLs. Genome Biol 13, R7, doi:10.1186/gb-2012-13-1-r7 (2012).

40 Johnson, A. D. et al. Polymorphisms affecting gene transcription and mRNA processing in pharmacogenetic candidate genes: detection through allelic expression imbalance in human target tissues. Pharmacogenet Genomics 18, 781-791, doi:10.1097/FPC.0b013e3283050107 (2008).

41 Nishida, H. et al. Histone $\mathrm{H} 3$ acetylated at lysine 9 in promoter is associated with low nucleosome density in the vicinity of transcription start site in human cell. Chromosome Res 14, 203-211, doi:10.1007/s10577-006-1036-7 (2006).

42 Millstein, J., Zhang, B., Zhu, J. \& Schadt, E. E. Disentangling molecular relationships with a causal inference test. BMC Genet 10, 23, doi:10.1186/1471-2156-10-23 (2009). 
43 Lambert, J. C. et al. Meta-analysis of 74,046 individuals identifies 11 new susceptibility loci for Alzheimer's disease. Nat Genet 45, 1452-1458, doi:10.1038/ng.2802 (2013).

44 Biological insights from 108 schizophrenia-associated genetic loci. Nature 511, 421-427, doi:10.1038/nature13595 (2014).

45 Gaulton, K. J. et al. Genetic fine mapping and genomic annotation defines causal mechanisms at type 2 diabetes susceptibility loci. Nat Genet 47, 1415-1425, doi:10.1038/ng.3437 (2015).

46 Finucane, H. K. et al. Partitioning heritability by functional annotation using genome-wide association summary statistics. Nat Genet 47, 1228-1235, doi:10.1038/ng.3404 (2015).

47 Karch, C. M., Ezerskiy, L. A., Bertelsen, S. \& Goate, A. M. Alzheimer's Disease Risk Polymorphisms Regulate Gene Expression in the ZCWPW1 and the CELF1 Loci. PLoS One 11, e0148717, doi:10.1371/journal.pone.0148717 (2016).

48 Del Villar, K. \& Miller, C. A. Down-regulation of DENN/MADD, a TNF receptor binding protein, correlates with neuronal cell death in Alzheimer's disease brain and hippocampal neurons. Proc Natl Acad Sci U S A 101, 4210-4215, doi:10.1073/pnas.0307349101 (2004).

49 Dourlen, P. et al. Functional screening of Alzheimer risk loci identifies PTK2B as an in vivo modulator and early marker of Tau pathology. Mol Psychiatry, doi:10.1038/mp.2016.59 (2016).

50 Westra, H. J. et al. Cell Specific eQTL Analysis without Sorting Cells. PLoS Genet 11, e1005223, doi:10.1371/journal.pgen.1005223 (2015).

51 Roeder, K., Devlin, B. \& Wasserman, L. Improving power in genome-wide association studies: weights tip the scale. Genet Epidemiol 31, 741-747, doi:10.1002/gepi.20237 (2007).

52 Chang, C. C. et al. Second-generation PLINK: rising to the challenge of larger and richer datasets. Gigascience 4, 7, doi:10.1186/s13742-015-0047-8 (2015).

53 Shi, Y. et al. Common variants on 8p12 and 1q24.2 confer risk of schizophrenia. Nat Genet 43, 1224-1227, doi:10.1038/ng.980 (2011).

54 Huang, L., Hu, F., Zeng, X., Gan, L. \& Luo, X. J. Further evidence for the association between the LSM1 gene and schizophrenia. Schizophr Res 150, 588-589, doi:10.1016/j.schres.2013.07.023 (2013).

55 lossifov, I. et al. The contribution of de novo coding mutations to autism spectrum disorder. Nature 515, 216-221, doi:10.1038/nature13908 (2014).

56 Bestman, J. E. \& Cline, H. T. The RNA binding protein CPEB regulates dendrite morphogenesis and neuronal circuit assembly in vivo. Proc Natl Acad Sci U S A 105, 20494-20499, doi:10.1073/pnas.0806296105 (2008).

57 Ruderfer, D. M. et al. Polygenic dissection of diagnosis and clinical dimensions of bipolar disorder and schizophrenia. Mol Psychiatry 19, 1017-1024, doi:10.1038/mp.2013.138 (2014). 